\title{
ACTIVE CONTROL OF A FLEXIBLE STRUCTURE USING \\ A MODAL POSITIVE POSITION FEEDBACK CONTROLLER
}

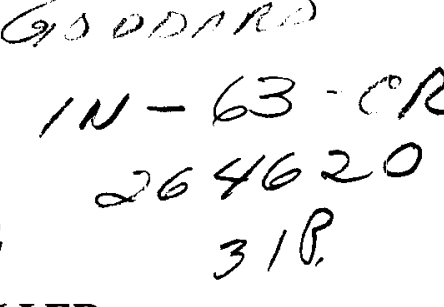

\author{
S.Poh and A.Baz \\ Mechanical Engineering Department \\ The Catholic University of America \\ Washington, DC 20064
}

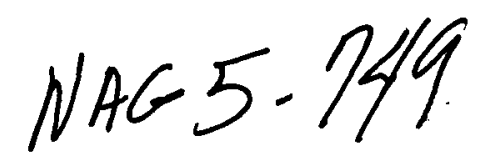

\section{ABSTRACT}

This study demonstrates the feasibility of a new Modal Positive Position Feedback (MPPF) strategy in controlling the vibration of a complex flexible structure using a single piezo-electric active structural member. The new control strategy generates its control forces by manipulating only the modal position signals of the structure to provide a damping action to undamped modes. This is in contrast to conventional modal controllers that rely in their operation on negative feedback of both the modal position and velocity. The proposed strategy is very simple to design and implement as it designs the controller at the uncoupled modal level and utilizes simple first order filters to achieve the Positive Position Feedback effect. The performance of the new strategy is enhanced by augmenting it with a "time sharing" strategy to share a small number of actuators between larger number of modes.

The effectiveness of the new strategy is validated experimentally on a flexible box-type structure that has four bays and its first two bending mode are 2.015 and $6.535 \mathrm{~Hz}$ respectively. A single piezo-electric actuator is utilized as an active structural member to control several transverse bending modes of the structure .

The performance of the active control system is determined in the time and the frequency domains. The results are compared with those obtained 
when using the Independent Modal Space Control (IMSC) of Meirovitch.

The experimental results suggest the potential of the proposed strategy as a viable means for controlling the vibration of large flexible structures in real time.

\section{Key Words}

Active control

piezo-electric actuators

Modal control

Positive Position feedback

time sharing 


\section{INTRODUCTION}

During the past few years, several active control systems have been successfully implemented to control actively the vibration of a wide variety of flexible structures. Such systems relied in their operation on different control algorithms that range from the simple velocity feedback control law [1-2] to the more imaginative methods such as the Independent Modal Space Control (IMSC) of Meirovitch [3-4] and the Positive Position Feedback (PPF) of Caughey and Goh [5] .

Attempts for validating these algorithms relied primarily on using voice-coil actuators in one and two way bay trusses at TRW [6] and CALTECH [7]. Recently, piezo-electric actuators have been gaining considerable acceptance as means for damping out the structural vibrations because of their high stiffness, as compared to the voice-coil actuators, their light weight, low power consumption as well as their wide frequency band. Attempts to utilize this class of actuators are numerous. Examples of such attempts include the work of Forward [8] who used ceramic piezo-actuators to damp out two closely spaced orthogonal bending modes in a cylindrical fiberglass mast. He utilized, in this regard, a simple rate damping control law . In 1985 and 1987, Hubbard and co-investigators [9-10] employed polymeric piezo-actuators (PVDF) to control the vibration of aluminum beams. Their control algorithm is of the distributed parameter type which is based on the application of Lyapunov's second method as devised by Kalmann and Bartram [11]. Crawley and De Luis [12-13] demonstrated also the effectiveness of using PZT actuators bonded as well as embedded in glass/epoxy and graphite/epoxy beams in attenuating the vibration of these composite beams. Simple one mode velocity feedback controller was used in these two studies.

In 1987, Fanson and Caughey utilized pairs of PVDF actuators/sensors to 
control the vibration of aluminum beams using a Positive Position Feedback algorithm [14]. Recently, Fanson et.al [15] incorporated piezo-electric pushers, as active members, in the JPL precision structure to control its flexible motions using Single Input - Single Output (SISO) control methods.

In 1988 and $1989, \mathrm{Baz}$ and co-workers [16-17] time shared a single ceramic piezo-actuator between several modes of vibrations of a flexible cantilevered beam using a Modified Independent Modal Space Control (MIMSC) method [18]. The MIMSC modifies the IMSC to account for the control spillover between the controlled and the uncontrolled modes. The MIMSC includes also a time sharing strategy to share small number of actuators between larger number of modes. Numerically [19] and experimentally [17], the MIMSC has been shown to have favorable vibration damping characteristics as compared to the IMSC and the Pseudo-Inverse (PI) method [17]. However, the MIMSC ,as other modal control methods, relies in its operation on feeding back both the modal position and velocity signals of the controlled modes to achieve the required vibration damping . Extraction of these signals from physical measurements is both time consuming and computationally intensive especially when dealing with large structures.

Therefore, it is the purpose of this study to demonstrate the effectiveness of the new strategy in obtaining stable and damped performance by positively feeding back the position signals in a manner similar to Caughey and Goh's Positive Position Feedback algorithm. However, five basic differences exist between the present study and that of Caughey and Goh's. First, the position signals are fed back, in this study, through first order filters instead of the second order filters of Caughey and Goh. This results in reducing the number of parameters required to design the controller to one third those needed to design the second order filters. Such 
simplification makes the selection of the optimal design parameters of the first order filters straight forward. This is contrast to the approximate and more complex pole-placement approach of Caughey and Goh which is based on "neglecting the coupling effect between the controlled and uncontrolled modes". Second, the controller is designed completely in the Independent Modal Space with the open-loop equations of the system remaining uncoupled even after including the modal controller. This is unlike Caughey and Goh's method where the originally uncoupled open-loop equations become coupled via the control law. Third, the present algorithm can be equally used in controlling the vibration and shape of flexible structures without producing any steady-state errors. Again this is not the case for the PPF method of Caughey and Goh. Fourth, the proposed algorithm results in uniform damping for all the modes as compared to Caughey and Goh's method where the damping ratio decreases continuously as the modal frequency increases. Fifth, a time sharing algorithm is used in the present study to share small number of actuators between larger number of modes. This feature was not addressed by Caughey and Goh.

These distinct differences, when coupled with the simplicity of the IMSC method, make the new control strategy easy to design and lend it effective in controlling the vibration as well as the shape of simple as well as large flexible structures. Such features have been successfully demonstrated by Baz, Poh and Fedor [20] when the new strategy is utilized to damp out two modes of vibration of a $25 \mathrm{~cm}$ long cantilevered beam using a single piezo-electric bimorph bonded to the beam.

In the present study the strategy will be extended and utilized to suppress the vibration of a box-type structure that has four bays and its 
first two bending modes occur at 2.015 and $6.075 \mathrm{~Hz}$. A single ceramic piezo-electric pusher is used, as an active structural member, to control simultaneously several modes of transverse vibrations.

The concept of the new control strategy and the selection of the optimal parameters of the first order filters are presented in section 2 . Section 3 includes the experimental evaluation of the strategy along with comparisons with the IMSC . Section 4 summarizes the conclusions of this study .

\section{THE MODAL POSITIVE POSITION FEEDBACK METHOD}

\subsection{The Concept}

The new method can be clearly understood by considering the block diagram shown in Figure (1). In the figure, the controller is used to control, in the independent modal space, the ith mode of an undamped flexible structure.

The controller feeds back positively the modal displacement $\mathbf{u}_{\mathrm{i}}$ through a first order filter that has a time constant $\tau_{i}$. The filter output $\mathbf{Y}_{\mathbf{i}}$ is added to the desired reference modal displacement $\mathbf{u}_{\mathrm{Ri}}$ and resulting signal is amplified by a proportional controller gain $\mathbf{K}_{\mathbf{i}}$. This gain is set equal to $\gamma_{i} \omega_{i}^{2}$, to be in a form similar to that of Caughey and Goh's, where $\omega_{i}$ is the natural frequency of the ith mode. The amplified signal $\mathbf{f}_{\mathbf{i}}$, i.e. the modal control action, is then sent to control the ith mode of the structure.

Mathematically, the interaction between the structural mode and the 


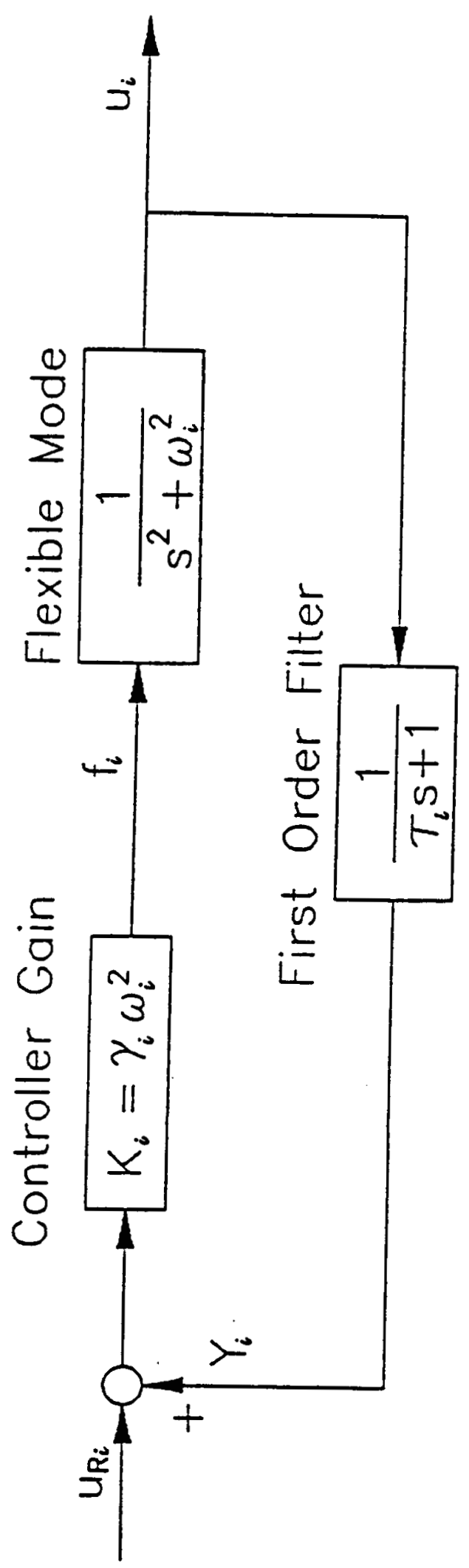

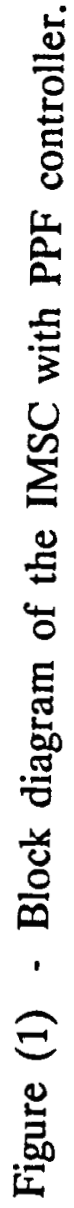


controller can be described as follows :

$$
\begin{array}{ll}
\text { The structure } & \ddot{u}_{i}+\omega_{i}^{2} u_{i}=f_{i}=\gamma_{i} \omega_{i}^{2}\left(Y_{i}+u_{R i}\right) \\
\text { The filter } & \tau_{i} \dot{Y}_{i}+Y_{i}=u_{i}
\end{array}
$$

\subsection{Stability condition}

The above structure-filter system has the following closed-loop transfer function

$$
\mathrm{u}_{\mathrm{i}} / \mathrm{u}_{\mathrm{Ri}}=\gamma_{\mathrm{i}} \omega_{\mathrm{i}}^{2}\left(\tau_{\mathrm{i}} \mathrm{s}+1\right) /\left[\tau_{\mathrm{i}} \mathrm{s}^{3}+\mathrm{s}^{2}+\tau_{\mathrm{i}} \omega^{2} \mathrm{~s}+\omega_{\mathrm{i}}^{2}\left(1-\gamma_{\mathrm{i}}\right)\right]
$$

where $\mathbf{s}$ is the Laplace operator. Applying Routh's stability criterion, it can be shown that the system is asymptotically stable for values of $\gamma_{i}<1$. Accordingly, it is possible for an undamped system to attain asymptotic stability by feeding positively its position signal through a simple first order filter without the need for any velocity feedback. This constitutes the basic premise of the present control algorithm.

\subsection{Zero steady-state condition}

The implementation of such modal control algorithm requires the selection of two design parameters which are namely : $\gamma_{\mathrm{i}}$ and $\tau_{\mathrm{i}}$. Actually, only the time constant $\tau_{\mathrm{i}}$ of the filter needs to be selected since $\gamma_{\mathrm{i}}$ must assume a fixed value $\gamma^{*}$ to eliminate the controller steady state error. This can be seen from equations (1) and (2) ,as the system attains its steady-state condition at time $t=\infty$ with $\dot{\mathrm{u}}_{\mathrm{i}}(\mathrm{t}=\infty)=0$ and $\dot{\mathrm{Y}}_{\mathrm{i}}(\mathrm{t}=\infty)=0$. Equation (1) yields

$$
\mathrm{u}_{\mathrm{i}}(\mathrm{t}=\infty)=\boldsymbol{\gamma}^{*}\left[\mathrm{Y}_{\mathrm{i}}(\mathrm{t}=\infty)+\mathrm{u}_{\mathrm{Ri}}\right]
$$

and equation (2) gives

$$
Y_{i}(t=\infty)=u_{i}(t=\infty)
$$


Eliminating $\mathrm{Y}_{\mathrm{i}}(\mathrm{t}=\infty)$ from equations (4) and (5) gives

$$
\left(1-\gamma^{*}\right) \mathrm{u}_{\mathrm{i}}(\mathrm{t}=\infty)=\gamma^{*} \mathrm{u}_{\mathrm{Ri}}
$$

For steady-state error to be zero, i.e. $u_{i}(t=\infty)=u_{R i}$, equation (6) requires that $\gamma^{*}=0.5$. Such a value is $<1$ and satisfies accordingly the asymptotic stability condition given in section $\mathbf{2 . 2}$. This zero steady-state condition is not satisfied in Caughey and Goh's method.

Therefore, the present algorithm can be equally used for shape control $\left(\mathrm{u}_{\mathrm{Ri}} \neq 0\right)$ and vibration control $\left(\mathrm{u}_{\mathrm{Ri}}=0\right)$.

\subsection{Optimal time constant of filter}

The optimal value of the time constant $\tau_{i}$ of the filter is determined by dividing the numerator and denominator of equation (3) by $\tau_{\mathrm{i}} \omega_{\mathrm{i}}^{3}$ to yield the following equation

$$
\begin{gathered}
\mathrm{u}_{\mathrm{i}} / \mathrm{u}_{\mathrm{Ri}}=0.5 \alpha(\overline{\mathrm{s}} / \alpha+1) /\left[\overline{\mathrm{s}}^{-3}+\alpha \overline{\mathrm{s}}^{2}+\overline{\mathrm{s}}+0.5 \alpha\right] \\
\alpha=1 / \tau_{\mathrm{i}} \omega_{\mathrm{i}} \text { and } \overline{\mathrm{s}}=\mathrm{s} / \omega_{\mathrm{i}}
\end{gathered}
$$

The above system has the following characteristic equation

$$
\overline{\mathrm{s}}^{3}+\alpha \overline{\mathrm{s}}^{2}+\overline{\mathrm{s}}+0.5 \alpha=0
$$

which has the root locus plot shown in Figure (2) for $0<\alpha<\infty$. The corresponding damping ratio $\zeta$ of the closed-loop system, as obtained from the root locus plot, is shown in Figure (3) as a function of $\alpha$ which is the only design parameter of the system. Figure (3) indicates that the damping ratio attains a maximum value of $20.07 \%$ when $\alpha=1.18$. This optimal value is very close to those obtained with the PPF method of Caughey and Goh as obtained experimentally by Fanson and Caughey [12]. Therefore, using first order filters, instead of the second order filters of Caughey and Goh, has simplified the design without compromising the damping characteristics of the 


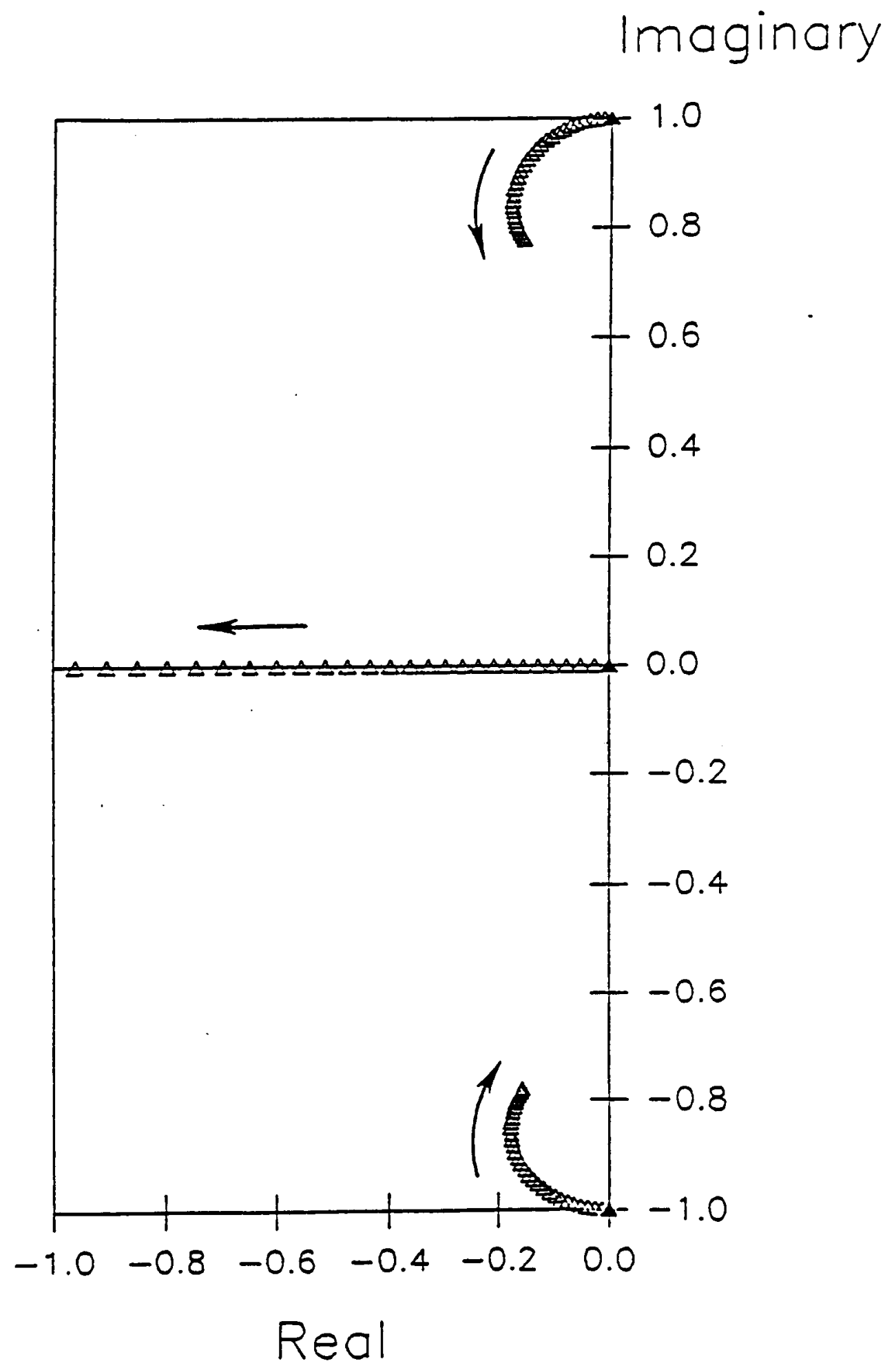

Figure (2) - Root locus of the IMSC with PPF Controller. 


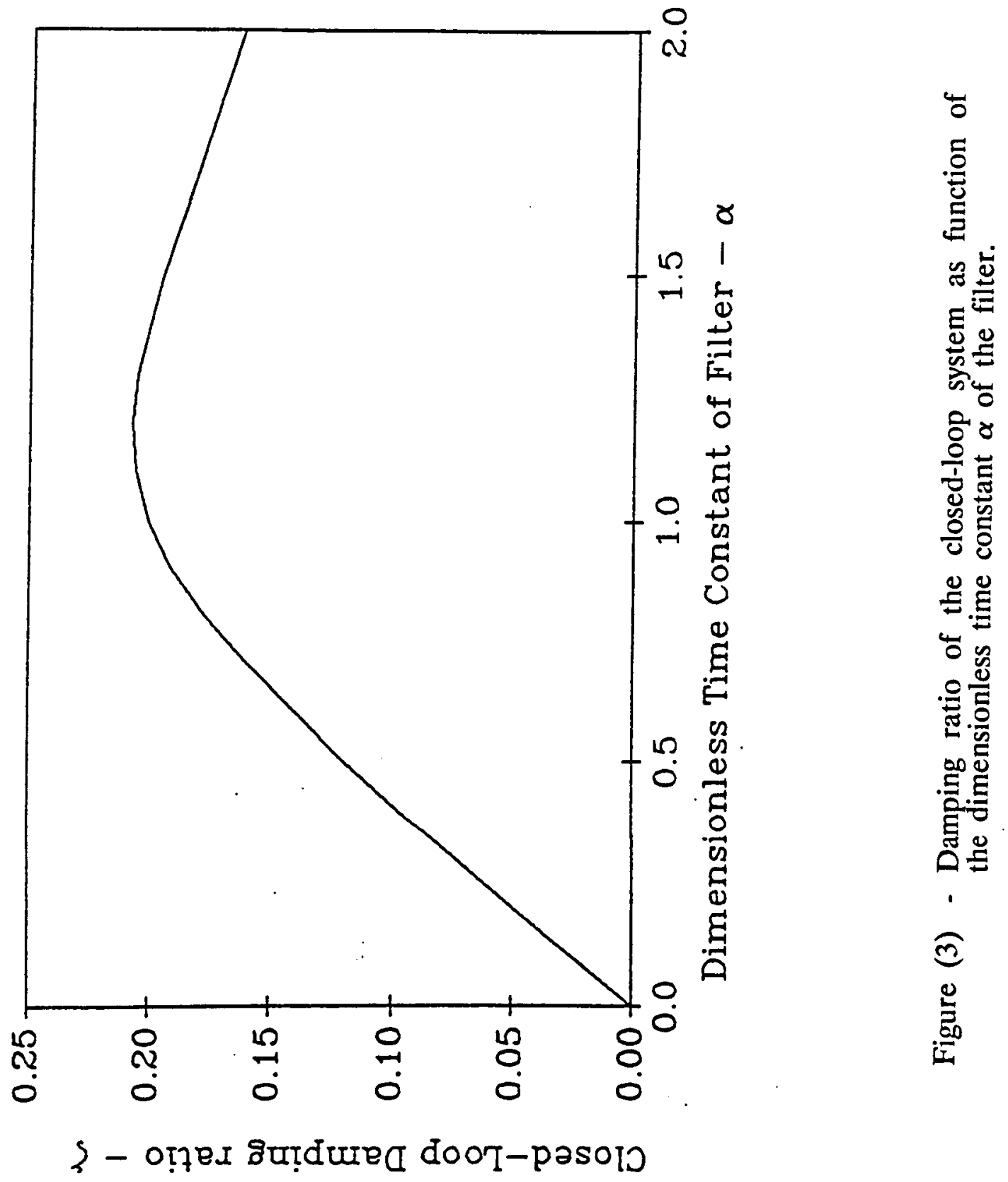


controller.

More importantly, the presented analysis being applicable to any mode, can result in uniform damping for all the modes if all their filters are tuned to satisfy the optimal tuning condition $(\alpha=1.18)$. Accordingly, a damping ratio of $20.07 \%$ can be maintained for any mode $\mathbf{i}$, that has natural frequency $\omega_{i}$, by selecting the time constant $\tau_{i}$ of its filter such that

$$
\tau_{\mathrm{i}}=1.18 / \omega_{\mathrm{i}} \quad \text { for } \mathrm{i}=1, \ldots, \mathrm{N}
$$

where $\mathrm{N}$ is the number of controlled modes.

Such uniform damping characteristics favors the present algorithm over that of Caughey and Goh's which has modal damping ratios that decrease continuously as the modal frequency increases.

Figure (4) shows a flow chart of the MPPF algorithm. The effectiveness of the algorithm in damping the vibration of flexible systems is validated experimentally in the what follows.

\section{EXPERIMENTAL VALIDATION OF THE ALGORITHM}

\subsection{THE TEST STRUCTURE}

Figure (5) shows a photograph of the test structure which is constructed to validate the developed control strategy. The structure is manufactured from polymethyl methacrylate rods that are $0.3125 \mathrm{~cm}$ in diameter which have Young's modulus and density of $4.2 \mathrm{GN} / \mathrm{m}^{2}$ and 3.32 $\left(\mathrm{gm} / \mathrm{cm}^{3}\right)$ respectively. The structure consists of four bays. The top three of which are configured in cubical form with $25 \mathrm{~cm}$ long longerons whereas the bottom bay, which is anchored to the base of support, is made from shorter longerons that are $17.25 \mathrm{~cm}$ long. Located in the bottom bay is the active 


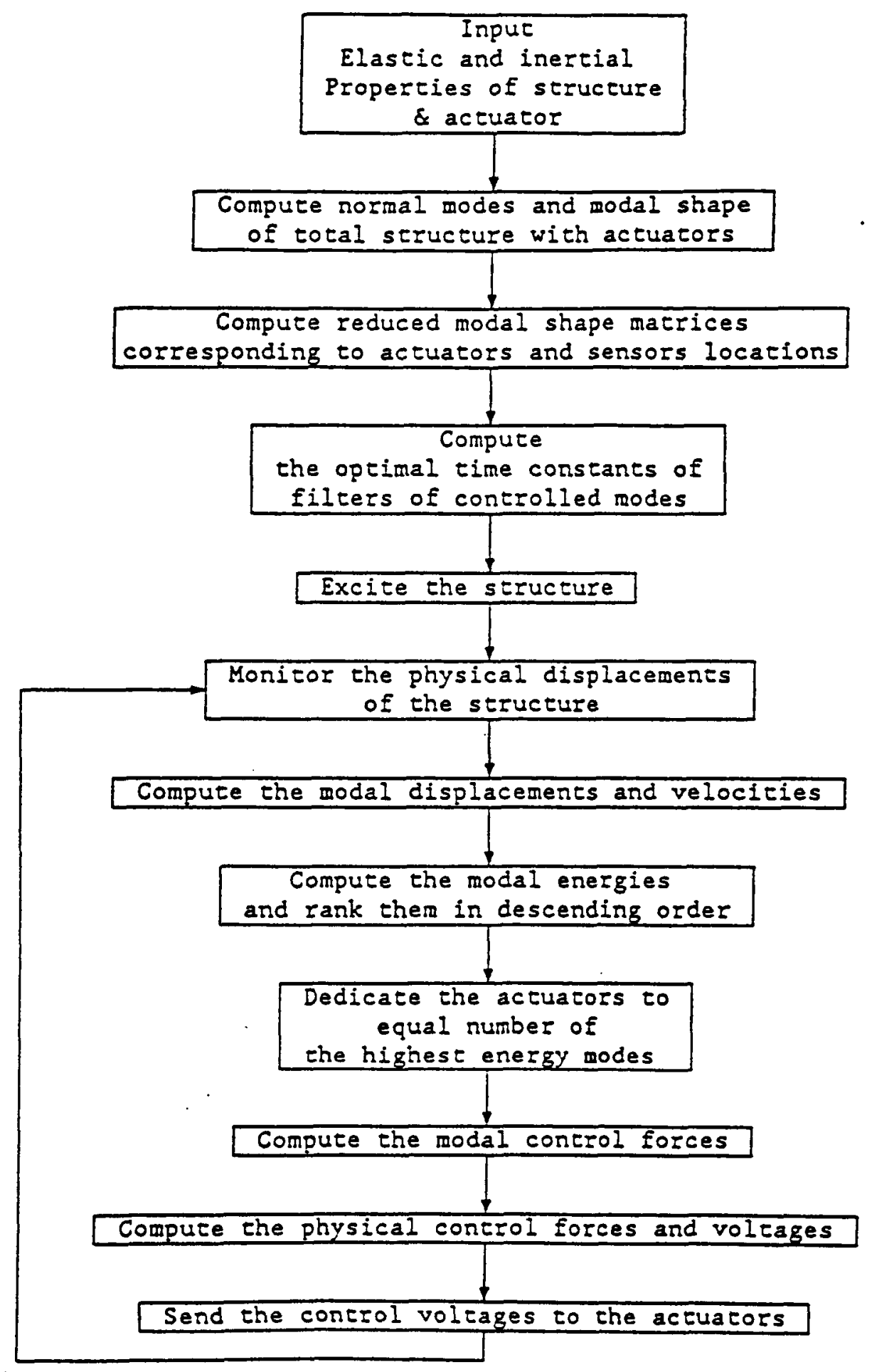

Figure (4) - Flow chart of the IMSC with PPF algorithm. 


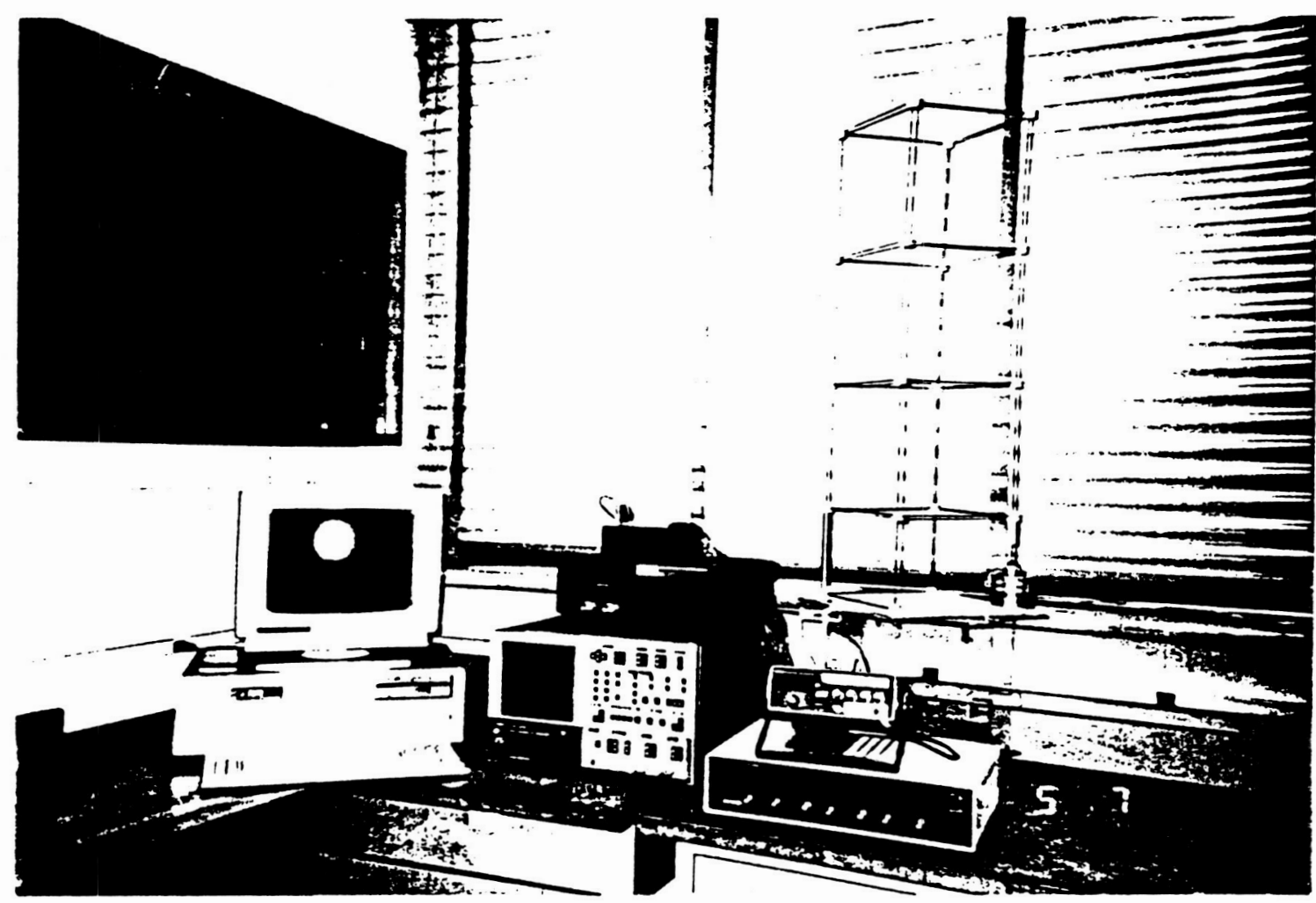

Figure (5) - Photograph of the test structure and associated controller and instrumentation. 
piezo-electric member as depicted in the photograph. A mechanical shaker is placed also in the bottom bay, diagonally opposite to the piezo-electric actuator, with its vibration axis coinciding with the longitudinal axis of the longeron. In this way the shaker is used to excite the structure axially as well as in the transverse flexural modes. The shaker is available commercially from Wilcoxon Research, Bethesda, MD 20814, USA (model F9/F3).

The first six modes of vibration of the structure as well as the corresponding modal shapes are obtained using the McNeal-Schwendler finite element package [21]. The results obtained are summarized in Figure (6). The figure indicates that the first and fourth modes, which are flexural modes in the $\mathrm{Y}$ directions, occur at 2.014 and $6.535 \mathrm{~Hz}$ respectively. Because of the structural symmetry, the second and fifth modes which correspond to transverse bending modes in the $\mathrm{Z}$ direction, occur also at 2.015 and 6.535 Hz. The torsion modes are found to be the third and the sixth modes which take place at 2.613 and $7.994 \mathrm{~Hz}$ respectively.

Experimental identification of the modes of transverse vibration of the structure is carried out using the impact hammer method [22]. The first and second modes, in the $\mathrm{Y}$ direction, are found to be 2.098 and $6.775 \mathrm{~Hz}$ respectively. These measurements deviate by 4.11 and $3.67 \%$ from the results calculated by the finite element method.

\subsection{The piezo-electric actuator}

The piezo-electric actuator, used in this study to control the vibration of the flexible structure, is made from stacked ceramic disks housed inside a stainless steel housing that is $16 \mathrm{~cm}$ long and $1.25 \mathrm{~cm}$ in diameter. The actuator is mounted between the structural nodes 1 and 2 as 
shown in Figure (6). Its main geometrical and physical characteristics are listed in Table (1). The actuator is available commercially from Burleigh Instruments , Burleigh Park, Fishers, N.Y., 14453, USA ( model PZL - 100) .

Table (1) - Main design parameters of the actuator

\begin{tabular}{|c|c|c|c|c|c|c|}
\hline $\begin{array}{c}\text { Stroke } \\
(\mu \mathrm{m})\end{array}$ & $\begin{array}{c}\text { Max } \\
\text { Volt }\end{array}$ & $\begin{array}{c}\text { Force } \\
(\mathrm{N})\end{array}$ & $\begin{array}{c}\text { Freq.Response } \\
(\mathrm{Hz})\end{array}$ & $\begin{array}{c}\text { Stiffness } \\
(\mathrm{N} / \mu \mathrm{m})\end{array}$ & $\begin{array}{c}\text { Hysteresis } \\
(\mathrm{8})\end{array}$ & $\begin{array}{c}\text { Iinearity } \\
(\mathrm{8})\end{array}$ \\
\hline 100. & 150 & 450 & 250 & 7.0 & 18 & 1.5 \\
\hline
\end{tabular}

The actuator is driven by a 386-based microprocessor which generates the necessary control action and feeds it through a D/A converter to a power amplifier (model PZ-350) which is also available commercially from Burleigh Instruments.

\subsection{The sensors}

Three non-contacting sensors are used to monitor the transverse vibrations of the structure at three nodal points 4,5 and 15 . The sensors are of the inductive type and are available commercially from Veeder-Root Co., Hartford, CT 06102, USA (model 576013-190). The signals of the three displacement sensors are sampled by the $\mathbf{3 8 6}$ micro-processor which is provided with a sampling and control board (model DASH-16) from METRA-BYTE corp., Tauton, MA 02780, USA. The board is capable of sampling 16 analog signals at a conversion time of $15 \mu \mathrm{s}$ with a resolution of 12 bits.

The physical signals of the sensors are used along with the modal shape matrix of the structure, obtained from the finite element analysis, to reconstruct the modal displacements of the structure. The dynamic condensation technique, of $\mathrm{M}$. Paz [23], is used to reduce the order of the structure to third order system which can be described completely in terms 

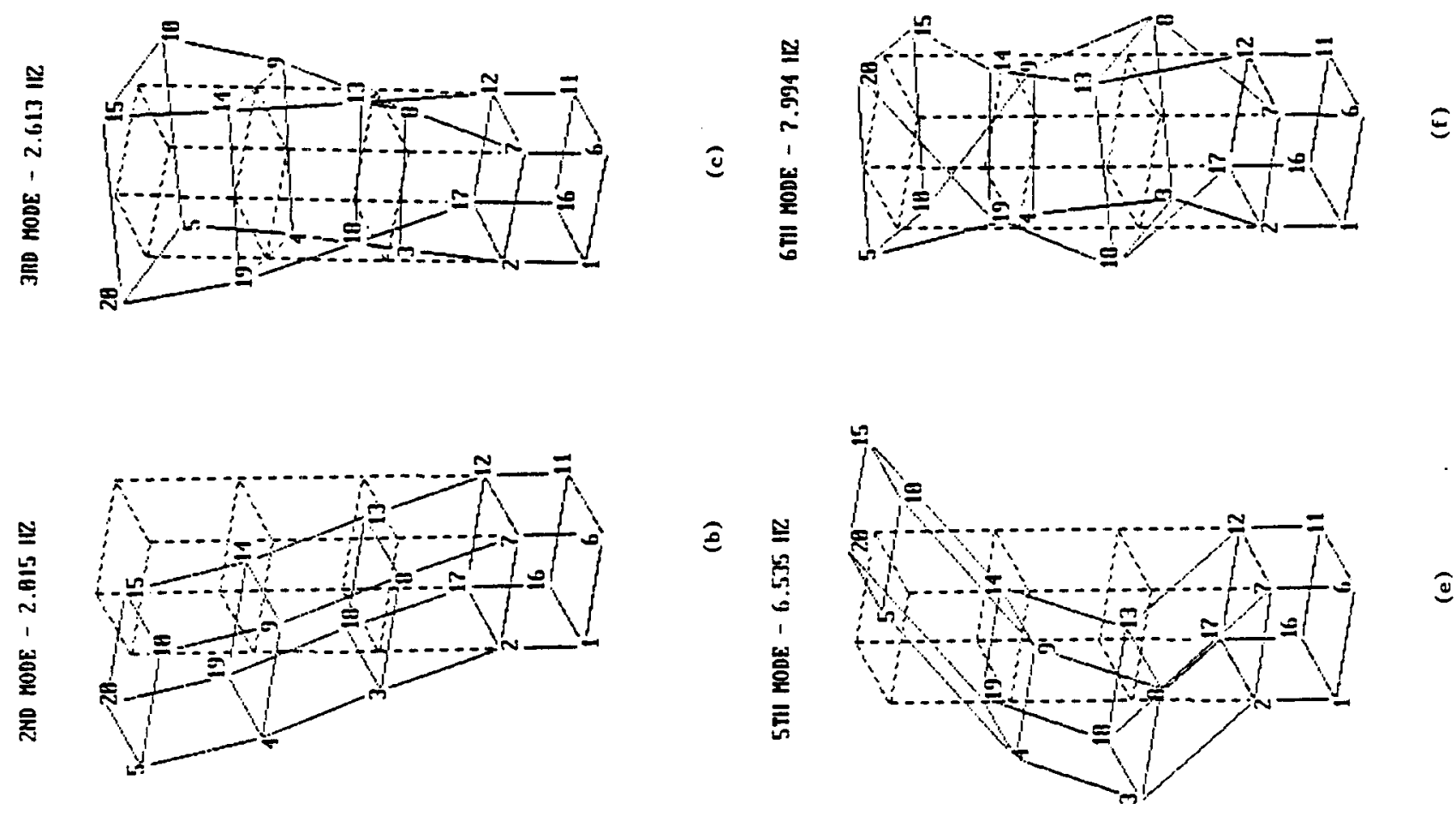

苛
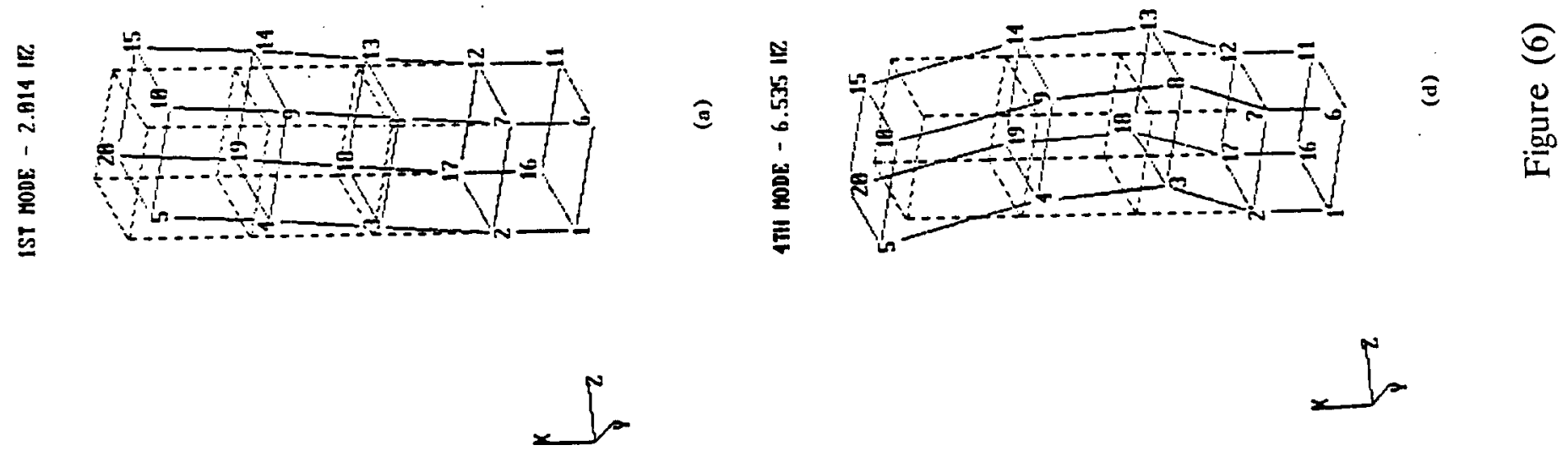
of the signals of the three sensors. The micro-processor uses the three sampled signals to compute the linear velocities of the structural nodes. The computed state variables $\left(\mathrm{y}_{4}, \mathrm{y}_{5}, \mathrm{y}_{15}, \dot{\mathrm{y}}_{4}, \dot{\mathrm{y}}_{5}\right.$ and $\left.\dot{\mathrm{y}}_{15}\right)$ are used to calculate the modal coordinates of the flexible system, the mode that has the highest modal energy, the corresponding optimal modal control force $\mathbf{f}_{\mathbf{i}}$, the physical control force $F_{c}$ and the necessary voltage $\mathbf{v}$ to be sent to the piezo-actuator. The implementation of these calculations, i.e. the MPPF algorithm, is carried out in real time in $2.52 \mathrm{~ms}$.

\subsection{Experimental results}

In all the experiments conducted in this study, the structure is excited near its second mode of vibration by driving the mechanical shaker at a frequency $7 \mathrm{~Hz}$. The excitations are maintained for a period of time until steady state conditions are attained. The shaker is then turned off and the structure is either left to vibrate freely (i.e.uncontrolled) or under the action of one modal control algorithm or another. The uncontrolled performance, shown in Figure (7-a), is used as a datum for judging the effectiveness of the different control algorithms.

Figures (7-b) and (7-c) show the time response of the structure when it is controlled by the new algorithm with the piezo-actuator dedicated either to the first mode alone or time shared between all the modes respectively. It is evident that the former approach is not as effective as the latter in damping out the structural vibration. In the time sharing approach, the actuator is dedicated to control the mode that has the highest instantaneous modal energy. This is not necessarily the lowest mode of vibration as it 

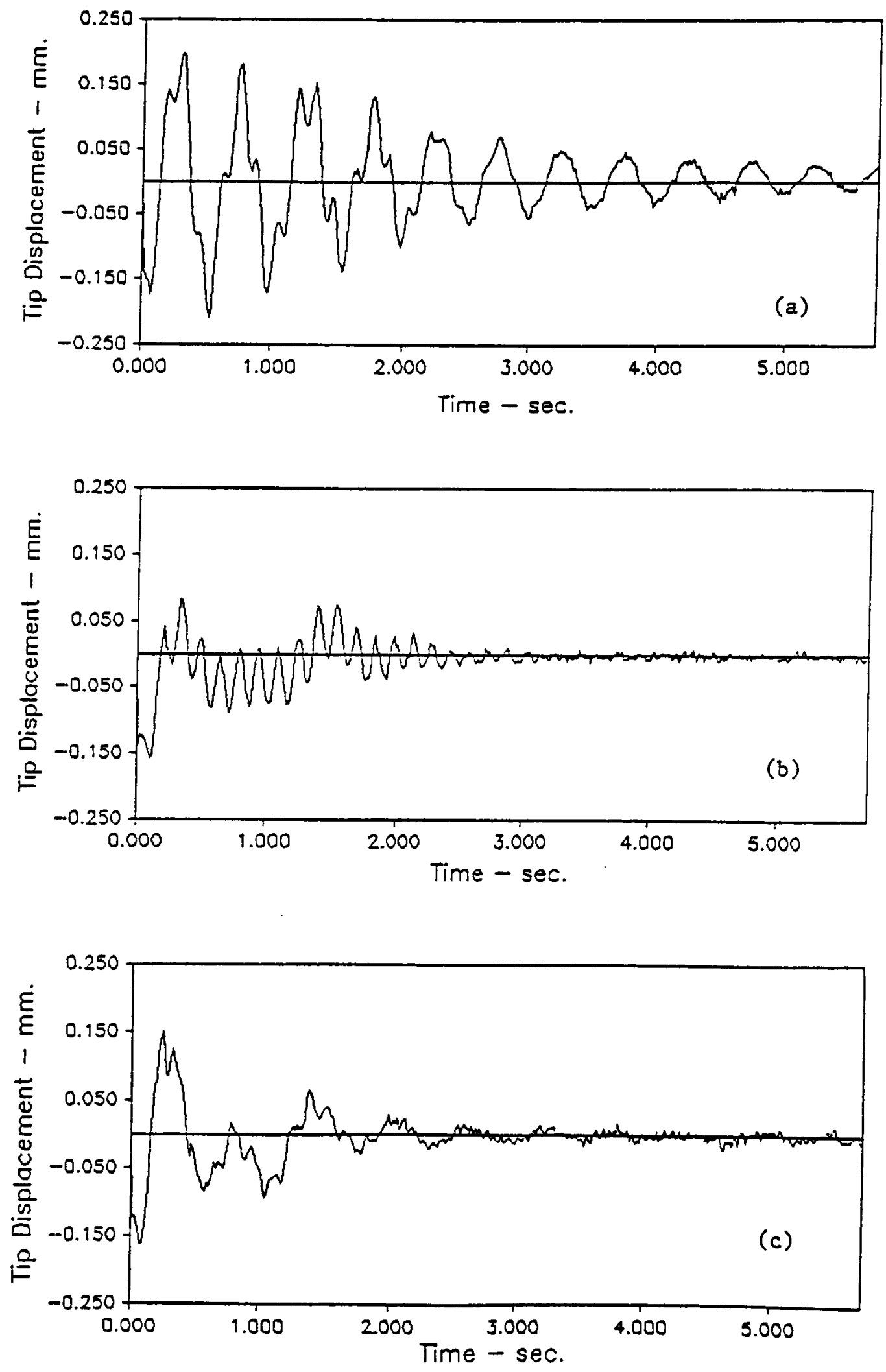

Figure (7) - Time response of structure (a. uncontrolled, b. controlled without time sharing and c. controlled with time sharing ). 
depends on the nature of the external disturbance. Figure (8) emphasizes clearly the fact that the mode that has the highest energy varies with time. As the controller suppresses one mode it excites others, at the same time, by virtue of the spillover effect. Accordingly, the effectiveness of the new algorithm, with its time sharing capability, stems from its adaptability to this continuously varying nature of the vibrating system. This effectiveness is demonstrated clearly in Figure (9) by considering the total modal energy of the structure when the actuator is dedicated to the lowest mode or time shared between the modes. It is clear that time sharing the single actuator between the modes results in reducing the modal energy faster than when it is dedicated to the lowest mode.

A better insight into the effectiveness of the new algorithm can be gained by considering the Fast Fourier Transform (FFT) of the structure response . Figure (10) shows the frequency content of the response of the uncontrolled structure in comparison with the controlled structure. These characteristics are obtained by sampling the position signal of the structure at node 15 by a spectrum analyzer and performing on it an FFT analysis. The figure emphasizes the effectiveness of the new algorithm particularly when it is provided with the time sharing capability.

Figure (11) shows comparisons between the time response of the uncontrolled structure and when it is controlled by the IMSC of Meirovitch and MPPF method. It is evident that the MPPF method is more effective than the IMSC method in damping out the vibration of the structure. This is in spite of the fact that the IMSC method uses both modal position and velocity signals to generate its control action. The IMSC method dedicates, however, the actuator to control the first mode of vibration which as 


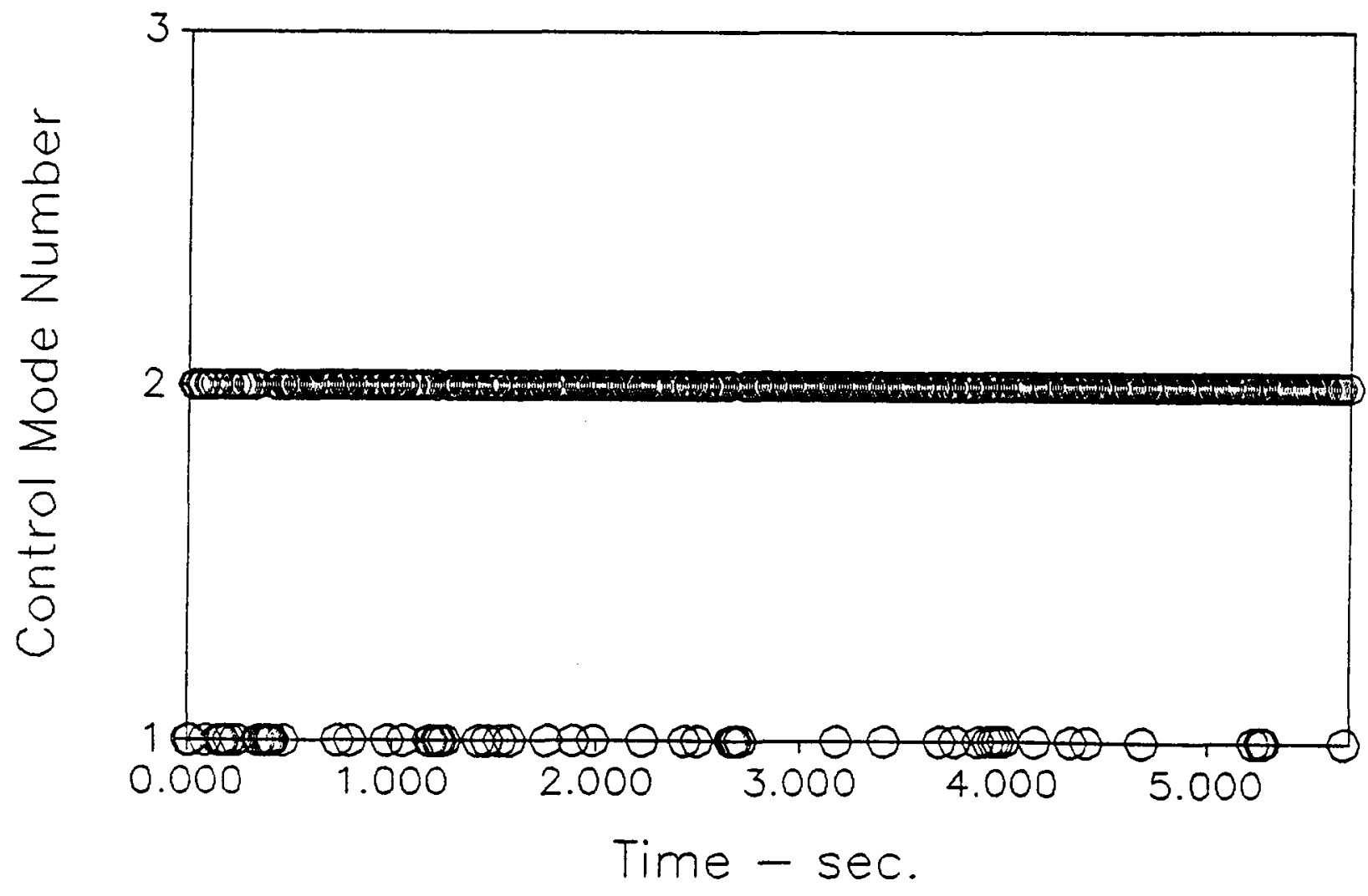

Figure (8) - The time history of the maximum modal energy mode 

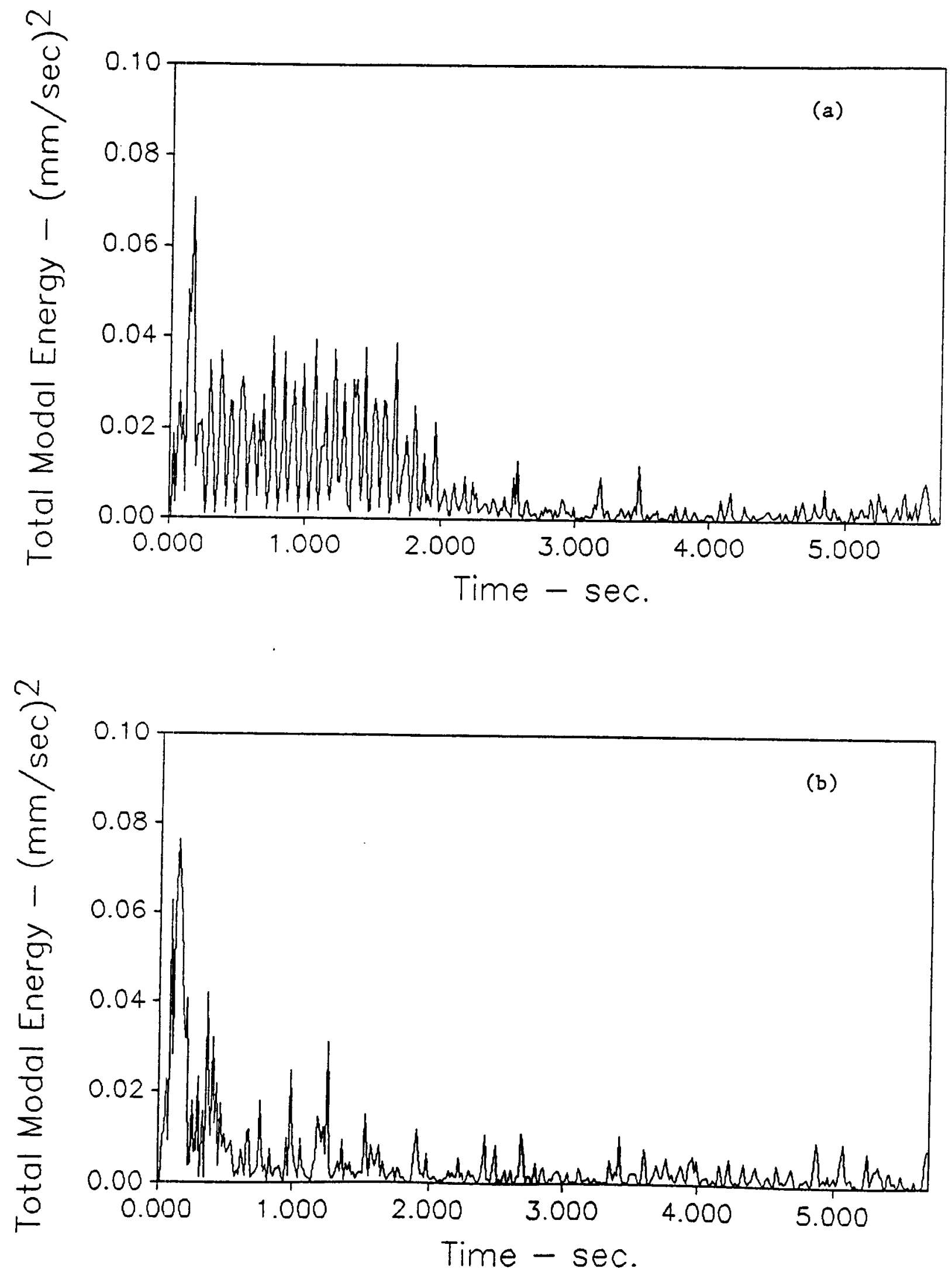

Figure (9) - The total modal energy of the structure (a. without time sharing, b. with time sharing ). 


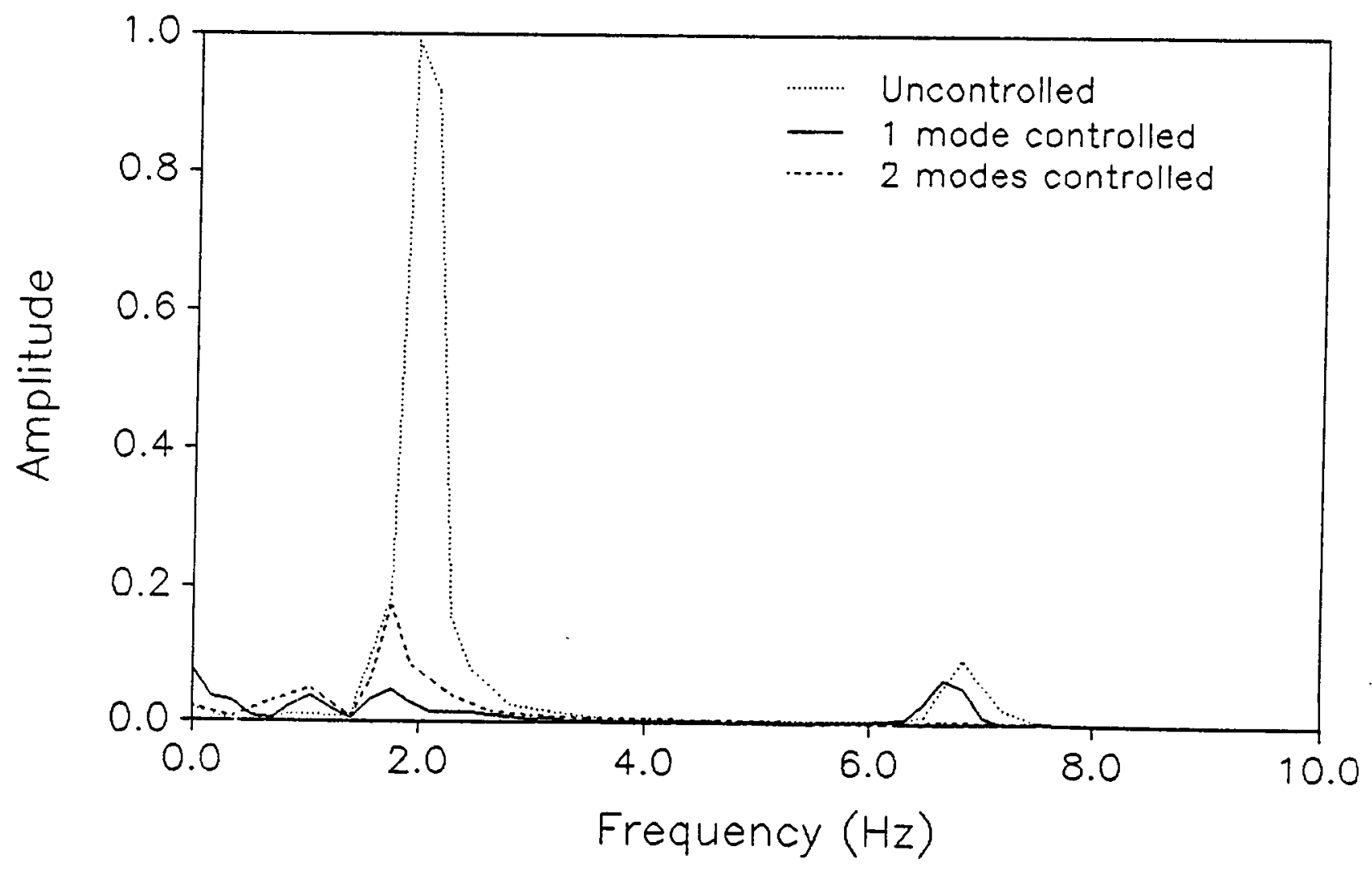

Figure (10) - Power spectrum of the displacement of node 15 of the structure (a. without time sharing b. with time sharing). 

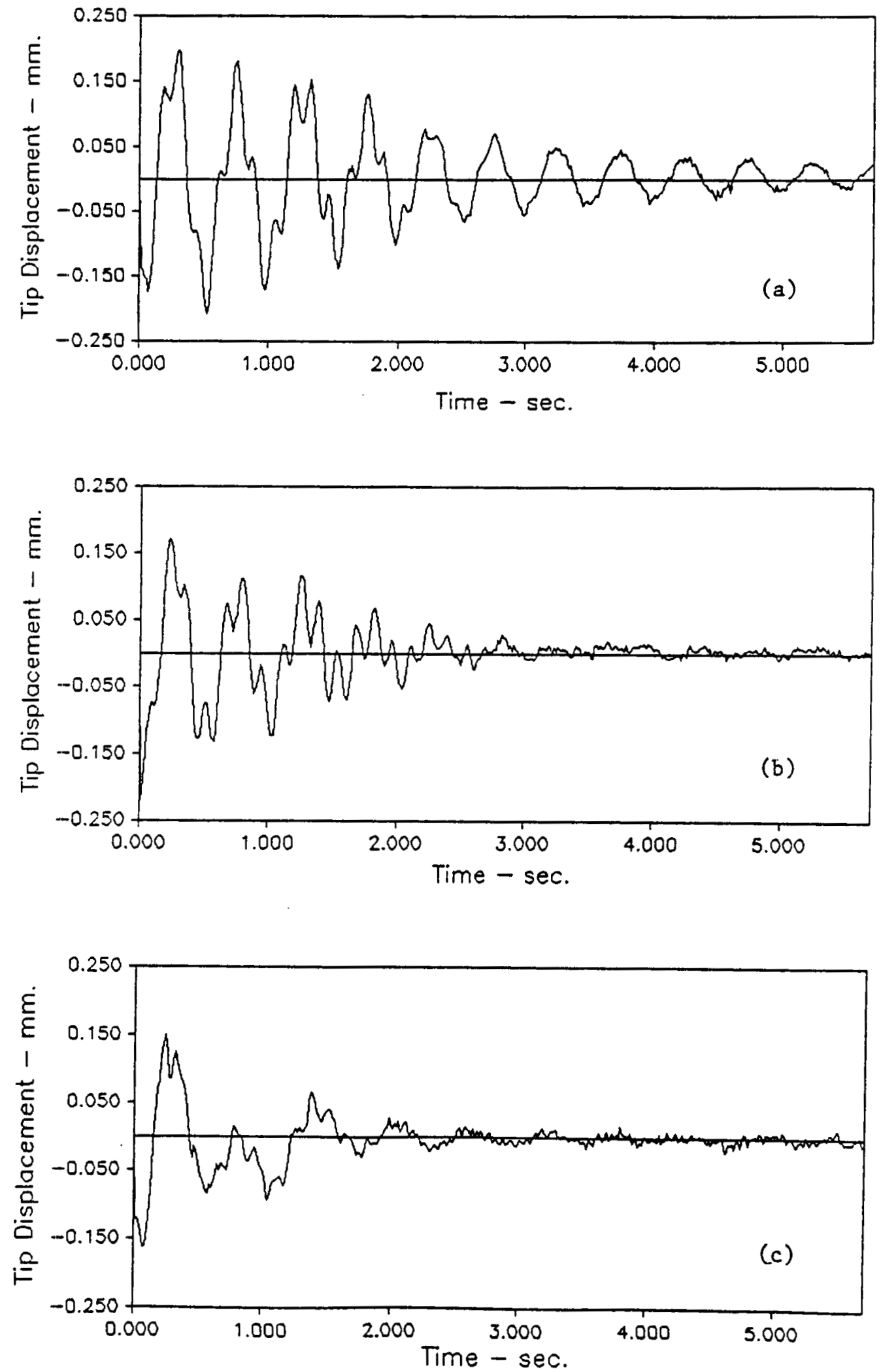

Figure (11) - Comparison between the time response of the structure (a. uncontrolled, b. with IMSC and c. with MPPF). 
mentioned above is not necessarily the mode that has the highest modal energy.

One should point out here that the IMSC method uses, however, less energy than the MPPF method to produce its control action as displayed in Figure (12).

\section{CONCLUSIONS}

This study has presented a new active control algorithm which is a combination of the IMSC and the PPF methods. The algorithm utilizes only modal position signals, fed through first order filters, to damp out the vibration of undamped flexible structures. The theory behind the algorithm is presented and the conditions for asymptotic stability and zero steady-state errors are derived. It is shown that uniform damping can be achieved for all the controlled modes if the filters are optimally tuned. The algorithm is validated experimentally using a single piezo-electric actuator to control the vibration of a flexible box-type structure. The results obtained indicate its effectiveness in suppressing structural vibration particularly when it is provided with time sharing capabilities. Comparisons ,carried out between the new algorithm and other modal control methods, emphasize its favorable damping characteristics.

The study demonstrates clearly the simplicity and potential of the method as an effective method for controlling large number of vibration modes with a smaller number of actuators. These features have important practical implications that make the algorithm invaluable means for controlling large space structures in real time. 

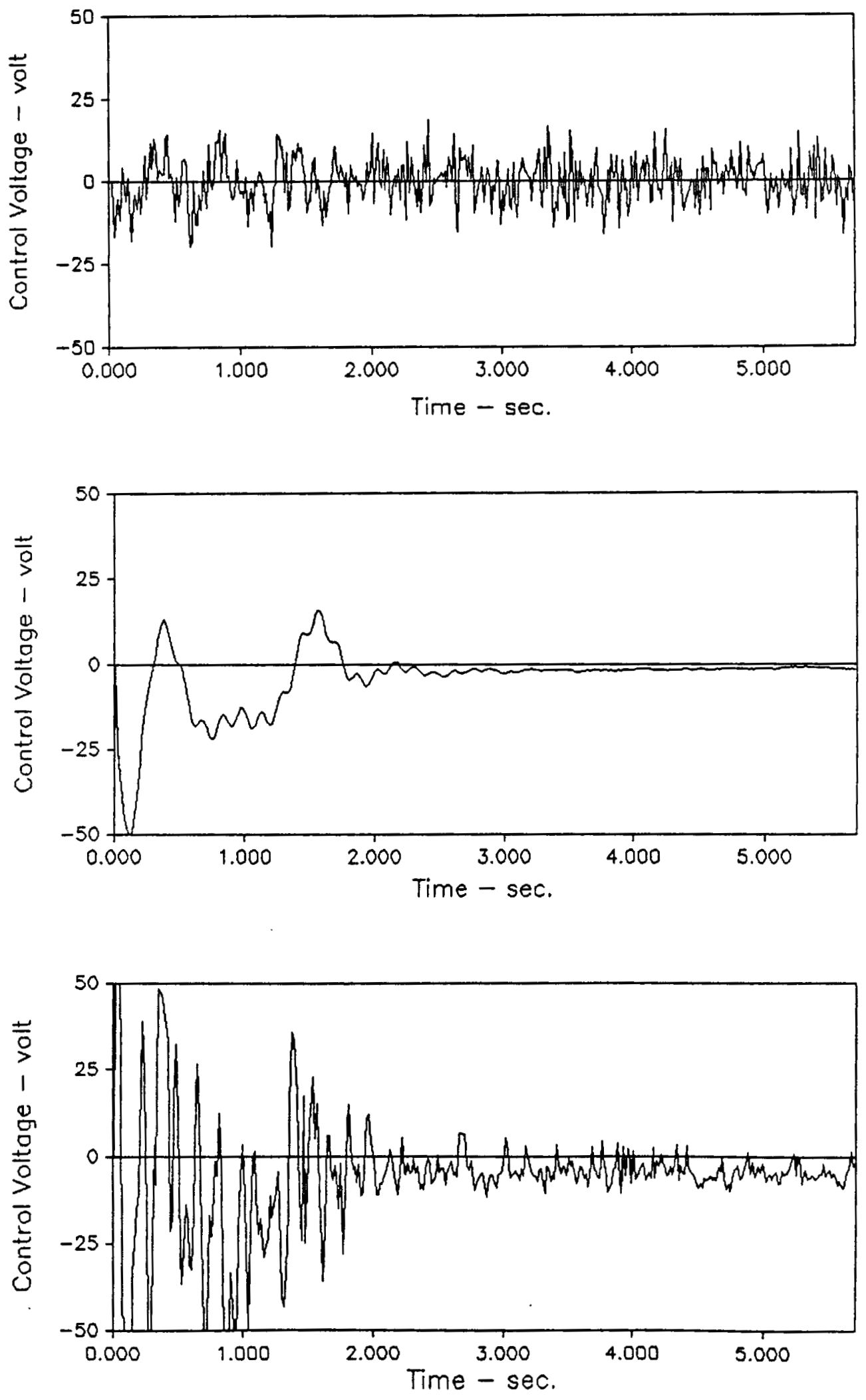

Figure (12) - Comparison between the control voltages of different methods (a. IMSC, b. MPPF without time sharing and c. MPPF with time sharing). 


\section{ACKNOWLEDGEMENTS}

This study was supported by NASA - Goddard Space Flight Center under grant number NAG 5-749. Special thanks are due to Dr.J.Fedor, the technical monitor, for his invaluable inputs and his thought stimulating discussions. 


\section{REFERENCES}

1. M.J.BALAS $1979 \mathrm{~J}$. of Guidance and Control 2, 252-253, Direct velocity feedback control of large space structures.

2. T.K.CAUGHEY AND C.J.GOH 1982 California Institute of Technology Report number DYNL-82-3. Analysis and control of quasi-distributed parameter systems.

3. L.MEROVITCH and H.BARUH 1981 Joumal of Guidance and Control 4 , 157-163. Optimal control of damped flexible gyroscopic systems.

4. L.MEIROVITCH and H.BARUH 1982 Joumal of Guidance and Control 5 , 59-66. Control of self-adjoint distributed-parameter systems.

5. C.J.GOH and T.K.CAUGHEY 1985 Int.J.Control, 41, 787-802. On the stability problem caused by finite actuator dynamics in the collocated control of large space structures.

6. L.R.DAILEY and M.LUKICH 1988 Proc. of The Automatic Control Conference.1468-1473. Atlanta, GA. Recent results in identification and control of flexible truss structure.

7. G.J.BALAS and J.C.DOYLE 1988 Proc. of The Automatic Control Conference . 1701-1702. Atlanta, GA. On the caltech experimental large structure.

8. R.L.FORWARD 1981 Journal of Spacecraft 18, 11-17. Electronic damping of orthogonal bending modes in a cylindrical mast.

9. T.BAILEY and J.E.HUBBARD, JR. 1985 Joumal of Guidance and Control 8, 605-611. Distributed Piezo-electric polymer active vibration control of a cantilever beam.

10. J.M.PLUMP, J.E.HUBBARD, JR. and T.Bailey 1987 ASME Journal of Dynamic Systems, Measurements and Control 109, 133-139. Nonlinear control of a distributed system: simulation and experimental results .

11. R.E.KALMANN and J.E. BARTRAM 1960 ASME J. of Basic Engineering, 371-400. Control system analysis and design via the 'second' method of Lyapunov.

12. E.F.CRAWLEY and J.de LUIS 1985 Proceedings of the 26th structures, structure Dynamics and Material Conference, Part 2, AIAA-ASME-ASCE, Orlando, Florida, 126-133. Use of piezo-ceramics as distributed actuators in large space structures.

13. E.F.CRAWLEY and J.de LUIS 1987 ALAA Journal 25, 1373-1385. Use of piezoelectric actuators as elements of intelligent structures.

14. J.L.FANSON and T.K.CAUGHEY 1987 ALAA paper number 87-0902. Positive position feedback control for large space structures.

15. J.L.FANSON, G.H.BLACKWOOD and C.C.CHU 1989 Proc. of 30th Structures, 
Structural Dynamics and Materials Conference. Part 3, AIAA-ASME-ASCE, Mobile, Alabama. 1480-1494.Active-Member Control of Precision Structures.

16. A.BAZ and S.POH 1988 Joumal of Sound and Vibration 126, 327-343. Performance of an active control system with piezoelectric Actuators.

17. A.BAZ and S.POH 1989 Joumal of Sound and Vibration (in press) Experimental implementation of the modified independent space control method.

18. A.BAZ, S.POH and P.STUDER 1989 Joumal of The Institution of Mechanical Engineers , Part C, 203, 103-112. Modified independent modal space control method for active control of flexible systems.

19. A.BAZ and S.POH 1987 NASA Technical report number N87.25605 Comparison between MIMSC, IMSC and PI in controlling flexible systems.

20. A.BAZ, S.POH and J.FEDOR 1989 Proc.of the 7th Conference on The Dynamics and Control of Large Structures. VPI \& SU, Blacksburg, VA. Independent modal space control with positive position feedback.

21. THE MacNEAL-SCHWENDLER CORP. 1987 Advanced Stress and Vibration Analysis, Version 3.0. Los Angeles, CA 90041.

22. D.J.EWINS 1984 Modal Testing : Theory and Practice. Letchwoth, England : Research Studies Press Ltd.

23. M.PAZ 1985 Structural Dynamics : Theory and Computation (2nd edition). New York : Van Nostrand Reinhold. 


\section{APPENDIX : NOMENCLATURE}

f modal force vector $(2 \mathrm{n} \times 1)$

$(\mathrm{N}, \mathrm{Nm})$

$f_{i} \quad$ controlled modal force of the ith mode

$(\mathrm{N}, \mathrm{Nm})$

$\mathrm{n} \quad$ number of nodal points

S Laplace operator

$u_{i} \quad$ modal displacement of the ith mode

$\dot{u}_{i} \quad$ modal velocity of the $i$ th mode

$\mathrm{u}_{\mathrm{Ri}} \quad$ reference modal displacement of the ith mode

v voltage applied across actuator (volts)

$y_{i} \quad$ linear translation of ith node

$Y_{i} \quad$ the output of the ith filter $(\mathrm{m}, \mathrm{rad})$

\section{Greek Symbols}

$\alpha \quad$ dimensionless time constant of filters

$\boldsymbol{\gamma}_{\mathrm{j}} \quad$ dimensionless gain of the controller

$\gamma^{*} \quad$ dimensionless gain of the controller for zero steadystate error $(=0.5)$

$\zeta_{i} \quad$ damping ratio of the ith mode

$\vartheta_{i} \quad$ angular deflection of ith node

$\lambda$ diagonal matrix of eigenvalues of the system (2nx2n)

$\tau_{\mathrm{i}} \quad$ time constant of the ith mode

$\omega_{i} \quad$ frequency of the ith mode 


\section{LIST OF FIGURES}

Figure (1) - Block diagram of the IMSC with PPF controller.

Figure (2) - Root locus of the IMSC with PPF Controller.

Figure (3) - Damping ratio of the closed-loop system as function of the dimensionless time constant $\alpha$ of the filter.

Figure (4) - Flow chart of the IMSC with PPF algorithm.

Figure (5) - Photograph of the test structure and associated controller and instrumentation.

Figure (6) - Modes of vibration of the test structure.

Figure (7) - Time response of structure ( a. uncontrolled, b. controlled without time sharing and c. controlled with time sharing).

Figure (8) - The time history of the maximum modal energy mode

Figure (9) - The total modal energy of the structure (a. without time sharing, b. with time sharing).

Figure (10) - Power spectrum of the displacement of node 15 of the structure (a. without time sharing b. with time sharing).

Figure (11) - Comparison between the time response of the structure (a. uncontrolled, b. with IMSC and c. with MPPF).

Figure (12) - Comparison between the control voltages of different methods (a. IMSC, b. MPPF without time sharing and c. MPPF with time sharing). 